\title{
ANÁlisis factorial de la Escala de Bienestar Psicológico de RyfF EN UNA MUESTRA DE UNIVERSITARIOS MEXICANOS
}

\author{
Maricela Osorio Guzmán y Carlos Prado Romero \\ Facultad de Estudios Superiores Iztacala \\ México
}

\begin{abstract}
RESUMEN
El bienestar psicológico es un constructo de interés para la psicología. El objetivo es analizar la validez de constructo de los principales modelos teóricos del bienestar propuestos en la literatura para determinar cuál presenta los niveles de ajuste más satisfactorios en población universitaria mexicana. Se aplicaron las escalas de bienestar psicológico (EBP, Riff, 1989a) a 1,400 estudiantes de 10 licenciaturas de la UNAM. Se probaron los modelos mediante un análisis factorial confirmatorio; se calculó la fiabilidad total y por áreas a partir de alfa de Cronbach y omega. Se encontraron dos estructuras de ajuste factorial satisfactorias, una de cuatro factores (17 reactivos y múltiples correlaciones) y otra de seis factores (20 ítems); con índices generales de fiabilidad adecuados. Se propone una versión válida y confiable de las escalas del bienestar para evaluar el constructo en universitarios mexicanos.
\end{abstract}

Palabras Clave:

Bienestar psicológico, propiedades psicométricas, análisis factorial confirmatorio, validez de constructo, estudiantes universitarios.

\section{Factorial analysis of the Ryff Psychological Well-being SCALE in A SAMPLE OF MEXICAN UNIVERSITY STUDENTS}

\begin{abstract}
Psychological well-being is a construct of interest for psychology. The aim was to analyze the construct validity of the main theoretical models of well-being proposed in the literature, in order to determine which one presents the most satisfactory levels of adjustment in the Mexican university population. The Psychological Well-Being Scales (EBP, Riff, 1989a) were applied to 1400 students from 10 UNAM degrees. The models were tested using a confirmatory factor analysis, total and area reliability were calculated from Cronbach's alpha and omega. Two satisfactory factorial adjustment structures were found, one with 4 factors (17 items and multiple correlations) and the other with 6 factors ( 20 items); with adequate general reliability indexes. A valid and reliable version of the well-being scales is proposed to evaluate the construct in Mexican university students.
\end{abstract}

Keywords:

psychological well-being, psychometric properties, confirmatory factor analysis, construct validity, university students.

BitÁCORA DEL ARTículo:

| Recibido: 28 de Julio de 2021 | Aceptado: 20 septiembre de 2022 | Publicado en línea: Enero - Junio de 2022 | 
Artículo Empírico | Análisis factorial de la Escala de Bienestar Psicológico... Osorio-Guzmán y Prado-Romero

Autoría y Derechos de Propiedad Intelectual

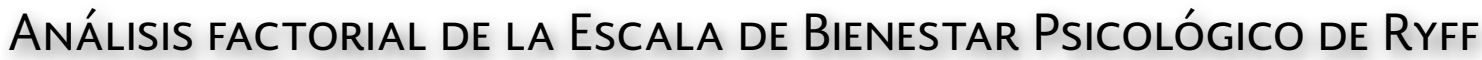 EN UNA MUESTRA DE UNIVERSITARIOS MEXICANOS
}

\author{
Maricela Osorio Guzmán y Carlos Prado Romero \\ Universidad Nacional Autónoma de México \\ México
}

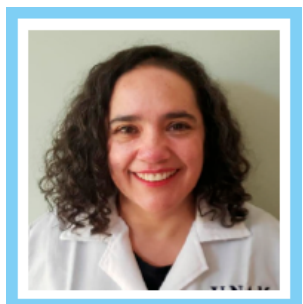

Maricela Osorio Guzmán FES- Iztacala, UNAM Correo: mosorio@unam.mx

Doctora en Ciencias Psicológicas y Pedagógicas con enfoque en Psicología de la Salud por la Universidad de los Estudios de Nápoles Federico II, (Italia). Es Profesor Titular "C" de la Carrera de Psicología UNAM. Miembro del Colegio de Docentes de Doctorado, en Italia; y del padrón de doctorado y maestría en la UNAM. Ha publicado en revistas especializadas nacionales e internacionales. Pertenece al Sistema Nacional de Investigadores (México). http://orcid.org/0000-0001-7798-5301.

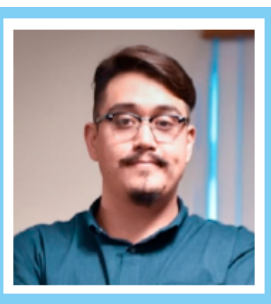

Carlos Prado Romero FES- Iztacala, UNAM

Correo: carlos.prado@iztacala.unam. $\mathrm{mx}$

Licenciado en Psicología. Doctorado en Psicología y Salud por la Universidad Nacional Autónoma de México. Profesor de Asignatura Ordinario "A" en la Facultad de Estudios Superiores Iztacala. Ha publicado en revistas especializadas nacionales e internacionales y participado en eventos académicos. https:// orcid.org/0000-0003-0809-0672

\section{CONTRIBUCIÓN DE LOS AUTORES}

Maricela Osorio Guzmán es responsable de la elaboración del marco teórico, aplicación de los instrumentos, análisis y redacción de datos, conclusiones y referencias. I Carlos Prado Romero estuvo a cargo de la elaboración del marco teórico, aplicación de los instrumentos, análisis y redacción de datos, conclusiones y referencias.

\section{AGRADECIMIENTOS}

Se agradece a todas aquellas personas que participaron e hicieron posible la realización de este estudio.

\section{Datos de Filiación de los Autores}

Facultad de Estudios Superiores Iztacala, UNAM

\section{(9) $(1)(0)$}

Copyright: (c) 2022 Osorio-Guzmán, M. \& Prado-Romero, C.

Este es un artículo de acceso abierto distribuido bajo los términos de la licencia Creative Commons Reconocimiento-NoComercial 4.0 Internacional, por lo que su contenido gráfico y escrito se puede compartir, copiar y redistribuir total o parcialmente sin necesidad de permiso expreso de sus autoras con la única condición de que no se puede usar con fines directamente comerciales y los términos legales de cualquier trabajo derivado deben ser los mismos que se expresan en la presente declaración. La única condición es que se cite la fuente con referencia a la Revista Digital Internacional de Psicología y Ciencia Social y a sus autoras. 


\section{TABLA DE CONTENIDO}

MÉTODO

Participantes, 5

Instrumentos, 5

Procedimiento, 5

Análisis de resultados, 5

RESUltados

Análisis de Modelos, 7

Análisis de fiabilidad, 8

Discusión

CONCLUSIONES

APÉNDICE

REFERENCIAS 
$\mathrm{E}$ bienestar es un constructo de interés para diversas disciplinas y áreas de la Psicología; con frecuencia este concepto se asocia con términos como felicidad, calidad de vida, salud psicológica, salud biológica, afectiva y familiar, así como con una buena situación económica (Buss, 2000; Costa, McCrae y Zonderman, 1987; Hagerty, 2000; Thompson, 2001; Casullo, 2002). En universitarios este constructo es relevante ya que frecuentemente se asocia con la percepción de apoyo social, así como con el logro académico, constructo que a su vez está relacionado con el esfuerzo y persistencia necesarios para el logro de objetivos y proyectos personales.

Este concepto se ha abordado desde perspectivas hedónicas y eudaimónicas. La primera implica satisfacción con la vida, presencia de estado de ánimo positivo, individual y específico en cada cultura, y se asocia sobre todo con el "bienestar subjetivo" (Diener, Suh, Luca y Smith, 1999; Kim-Prieto, Diener, Tamir, Scollon y Diener, 2005; García-Alandete, 2013; Vielma y Alonso, 2010). La segunda implica la realización del potencial individual, el crecimiento personal y la actualización de las capacidades como evidencia de funcionamiento psicológico positivo (Keyes, 2006; Ring, Höfer, McGee, Hickey y O'Boyle, 2007; Ryan y Huta, 2009).

Una de las autoras principales de la teoría del bienestar psicológico es Ryff (1989a), quien lo concibió desde claves eudaimónicas, y propuso el "Modelo Multidimensional del Bienestar Psicológico", identificando seis dimensiones constituyentes: autoaceptación (evaluación positiva de uno mismo y la propia vida pasada), relaciones positivas con otras personas (desarrollo y mantenimiento de relaciones de calidad y confianza con otros), autonomía (sentido de autodeterminación personal), dominio del entorno (capacidad de dirigir efectivamente la propia vida y el ambiente para satisfacer necesidades y deseos), propósito en la vida (creencia de que la propia vida tiene un propósito y un significado) y crecimiento personal (sentido de crecimiento continuo y desarrollo como persona); partiendo de este modelo, la autora diseñó las Escalas de Bienestar Psicológico (Psychological Well-Being Scales [PWBS]; Ryff, 1989a, 1989b; Ryff y Singer, 2008; Keyes, Shmotkin y Ryff, 2002; García-Alandete, 2013; Ryan y Huta, 2009).

Esta escala es uno de los instrumento de evaluación del bienestar más utilizado en la investigación; se ha usado en diferentes países en muestras etarias e idiomas distintos, y en múltiples versiones en cuanto a la cantidad de ítems (Abbott, Ploubidis, Huppert, Kuh y Croudace, 2010; Abbott et al., 2006; Freire, Ferradás, Nuñez, y Valle, 2017; García-Alandete, 2013; Loera, Balcázar, Trejo, Gurrola, Bonilla, 2008; Medina, Gutiérrez y Padrós, 2013; Tomás, Meléndez y Navarro, 2008; Veliz, 2012; Vera-ViIlarroel, Urzúa, Silva, Pavez y Celis, 2013).

A pesar de su extenso uso, la validez de este modelo ha sido puesta a prueba en múltiples ocasiones debido a que las soluciones factoriales varían de modo considerable, generando nuevas preguntas y análisis minuciosos (Freire et al., 2017; Springer y Hauser, 2006; Tomás et al., 2008; Van Dierendonck, Díaz, Rodríguez-Carvajal, Blanco y Moreno-Jiménez, 2008; Vera-Villarroel et al., 2013). De acuerdo con Freire et al. (2017), Ryff también plantea varias posibilidades e índices de ajuste.

De esta manera, entre los modelos propuestos está el de seis dimensiones de primer orden con un factor latente de segundo orden, con algunos arreglos específicos (Ryff y Keyes, 1995), como uno de los modelos con mejor ajuste y que ha sido comprobado por diversas investigaciones (Abbott et al., 2006; Chen, Jing, Hayes y Lee, 2013; Díaz et al., 2006; Kállay y Rus, 2014; Tomás et al., 2008; Van Dierendonck, 2004). También hay otro modelo donde se plantea que, en la estructura de seis factores de primer orden, se encontrarían dos constructos de nivel superior (bienestar subjetivo y bienestar psicológico) (Keyes et al., 2002). En otras investigaciones, autores como Tomás, Sancho, Meléndez y Mayordomo (2012) obtuvieron buenos índices de ajuste al eliminar dos dimensiones (relaciones positivas con otros y autonomía) o con un modelo de cinco factores (Kafka y Kozma, 2002; Tomás et al., 2008); por su parte, Rodríguez, Díaz, Moreno, Blanco, y Van Dierendonk (2010) propusieron un modelo que incluye ocho dimensiones.

Debido al interés de esta investigación y a que existen pocos estudios que prueban diferentes modelos estructurales del bienestar psicológico en población universitaria (Freire et al., 2017); debido a que este constructo resulta de especial interés en esta muestra por las particularidades que les caracterizan (por ejemplo, su rendimiento es constantemente evaluado, tienen periodos amplios de clase, muchas horas de estudio, diseño de proyectos y tareas, relaciones conflictivas con docentes y coetáneos y/o poco apoyo familiar (Caballero, Bresó y González, 2015; Carlotto, Gonçalves-Câmara y Brazil-Borges, 2005; Martínez y Márques, 2005), y por las problemáticas que han sido reportadas (niveles de bienestar bajo y moderado, niveles menores de bienestar en hombres, niveles de bienestar dependiendo de la sede universitaria (Ross-Argüelles, Salgado-Ortiz, Fernández-Nistal y López-Valenzuela, 2019; Zubieta y 
Delfino, 2010; Osorio, Prado, Bazán y Parrello, 2021), y porque al momento de la presente investigación sólo se encontraron tres artículos (Medina et al., 2013; Loera et al., 2008; Jurado-García, Benítez-Hernández, Mondaca-Fernández, Rodríguez-Villalobos y Blanco-Ornelas, 2017) y un capítulo de libro (Lozano y García, 2016) que examinan las características psicométricas de la escala en México — sin analizar los modelos estructurales subyascentes con diferente número de reactivos-, es que se torna imperante analizar la estructura factorial de la Escala de Bienestar en universitarios del país.

Por lo referido, en el presente estudio se analizó la validez de constructo de los principales modelos estructurales teóricos del bienestar propuestos en la literatura, para determinar cuál de ellos presenta los niveles de ajuste más satisfactorios para población mexicana universitaria.

\section{MÉTOdo}

\section{Participantes}

400 estudiantes con una media de edad de 20.58 años (DE $=2.1$ ), matriculados en 10 carreras universitarias impartidas en cinco campus de la Universidad Nacional Autónoma de México (Ciudad Universitaria, 31.8\%; FES Acatlán, 11.5\%; FES Aragón, 24.3\%; FES Iztacala, 23.6\%, y FES Zaragoza, $8.9 \%$ ); el semestre en que la mayoría estaba inscrito fue el cuarto; $48.1 \%$ hombres y $51.9 \%$ mujeres.

\section{Instrumentos}

Para evaluar el bienestar psicológico se aplicaron las Escalas de Bienestar Psicológico de 39 reactivos (22 directos y 17 indirectos) con opciones de respuesta en escala tipo Likert de seis puntos $(1=$ totalmente en desacuerdo a $6=$ totalmente de acuerdo) en su versión al español (Díaz et al., 2006), que evalúa los seis factores descritos por Ryff (1989a): Autoaceptación (seis reactivos, $\alpha=0.83$ ), Relaciones positivas (seis reactivos, $\alpha=0.81$ ), Autonomía (ocho reactivos, $\alpha=0.73$ ), Dominio del entorno (seis reactivos, $\alpha$ $=0.71$ ), Propósito en la vida (siete reactivos, $\alpha=0.83$ ), Crecimiento personal (seis reactivos, $\alpha=0.68$ ), donde a mayor puntuación, mayor nivel de bienestar psicológico.

\section{Procedimiento}

El presente trabajo de investigación se efectuó siguiendo los lineamientos éticos para el trabajo e investigación con personas de la Sociedad Mexicana de Psicología (SMP, 2002), así como la Declaración de Helsinki 1995 y modificaciones posteriores.

El levantamiento de datos fue hecho en un lapso de cuatro semanas mediante un muestreo no probabilístico por conveniencia, a través de un formulario de Google Forms distribuido por redes sociales, en el cual se invitó a participar a la comunidad universitaria en la investigación. El Formato contenía un consentimiento informado que indicaba que la participación era voluntaria y anónima, que todos los datos recopilados serían procesados de manera agregada y se garantizaba el anonimato de los participantes; por otra parte, se indicó que los mismos se almacenarían por un año con el uso de medios tecnológicos como contraseñas cifradas, acceso a los datos sólo por personal autorizado, etcétera.

Los estudiantes que aceptaron participar en la investigación (marcando la opción correspondiente en el formulario) accedieron al instrumento y procedieron a responderlo.

Una vez completado el levantamiento de datos se procedió a la construcción y limpieza de la base de datos.

\section{Análisis de resultados}

En primer lugar se efectuó la limpieza de la base de datos haciendo un escaneo para detectar valores perdidos y respuestas no comprometidas, eliminando los casos con índices superiores a 5\% de valores perdidos sobre el total de variables y desviaciones estándar inferiores a 0.5 y/o superiores a valores $Z=3$, según lo indicado por Streiner, Norman y Cairney (2015).

Luego se hizo una división aleatoria de la base de datos en dos submuestras homogéneas independientes (50\% cada una). La primera para efectuar los análisis factoriales confirmatorios (AFC) de los principales modelos de bienestar psicológico obtenidos en diferentes investigaciones $(n=700)$; la segunda se utilizó para comprobar los modelos identificados como los más adecuados, y establecer las covarianzas especificadas como índices de modificación propuestos por el programa estadístico; lo anterior se retoma del tratamiento estadístico hecho por Freire et al. (2017).

Los modelos probados son seis modelos propuestos por Díaz et al. (2006).

El modelo 1 postula la existencia de un único factor etiquetado como bienestar psicológico, en el que se agrupan todos los ítems.

El modelo 2 muestra una distribución bifactorial donde los ítems positivos $(1,3,6,7,10,11,12,14,16$, $17,18,19,21,23,24,28,31,32,35,37,38,39)$ saturan en un factor, y los ítems negativos $(2,4,5,8,9,13$, $15,20,22,25,26,27,29,30,33,34,36)$ en otro.

El modelo 3 sugiere una composición de cinco dimensiones donde los reactivos de las escalas de autoaceptación y dominio del entorno se agrupan en un factor $(1,7,13,19,25,31,5,11,16,22,28,39)$, relaciones positivas con otras personas $(2,8,14,20,26,32)$, auto- 
nomía $(3,4,9,10,15,21,27,33)$, propósito en la vida $(6,12,17,18,23,29)$ y crecimiento personal $(24,30$, $34,35,36,37,38)$ (Kafka y Kozma, 2002).

El modelo 4 representa la estructura original de seis dimensiones descritas por la autora del instrumento: autoaceptación $(1,7,13,19,25,31)$, relaciones positivas con otras personas $(2,8,14,20,26,32)$, autonomía $(3,4,9,10$, $15,21,27,33)$, dominio del entorno $(5,11,16,22,28,39)$, propósito en la vida $(6,12,17,18,23,29)$ y crecimiento personal $(24,30,34,35,36,37,38)$ (Ryff, 1989a , 1989b).

El modelo 5 expone una estructura de cinco factores donde los ítems de las escalas de autoaceptación y dominio del entorno se agregan a un único factor, junto con un factor de segundo orden denominado bienestar psicológico (autoaceptación [1, 7, 13, 19, 25, 31], dominio del entorno $[5,11,16,22,28,39])$, relaciones positivas con otras personas $(2,8,14,20,26,32)$, autonomía $(3,4,9$, $10,15,21,27,33)$, propósito en la vida $(6,12,17,18,23$, 29) y crecimiento personal $(24,30,34,35,36,37,38)$.

El modelo 6 representa la estructura propuesta por Van Dierendonck (2004), quien propone añadir a la estructura original un factor de segundo orden, al cual denominó bienestar psicológico (autoaceptación [1, 7, 13, $19,25,31]$, relaciones positivas con otras personas [2, 8, 14, 20, 26, 32], autonomía [3, 4, 9, 10, 15, 21, 27, $33]$, dominio del entorno [5, 11, 16, 22, 28, 39], propósito en la vida $[6,12,17,18,23,29]$ y crecimiento personal $[24,30,34,35,36,37,38])$.

Los modelos probados por Freire et al. (2017). El modelo 7 representa el diseño de Abbott et al. (2010), donde el bienestar está determinado por tres factores: 1$)$ relaciones positivas $(2,8,14,20,26,32)$; $)$ autonomía $(3,4,9$, $10,15,21,27,33)$, y 3$)$ un factor latente de segundo orden que comprende las dimensiones autoaceptación (1, $7,13,19,25,31)$, dominio del entorno $(5,11,16,22,28$, $39)$, propósito en la vida $(6,12,17,18,23,29)$ y crecimiento personal $(24,30,34,35,36,37,38)$.

El modelo 8 está conformado por cuatro dimensiones: autoaceptación $(1,7,13,19,25,31)$, dominio del entorno $(5,11,16,22,28,39)$, propósito en la vida (6, $12,17,18,23,29)$ y crecimiento personal $(24,30,34$, 35, 36, 37, 38) (Tomás et al., 2012).

El modelo 9 propone un modelo de cuatro dimensiones y un constructo latente de segundo orden (bienestar psicológico), que incluye las dimensiones de autoaceptación $(1,7,13,19,25,31)$, dominio del entorno $(5,11,16,22,28,39)$, propósito en la vida $(6,12,17$, $18,23,29)$ y crecimiento personal $(24,30,34,35,36$, $37,38)$.

Así como el modelo emanado de sus datos. El modelo 10 (Freire et al., 2017) propone un modelo de cua- tro factores, retomando sólo 17 ítems distribuidos como sigue: autoaceptación $(7,17,24)$; dominio del entorno $(5,10,14,19,29)$ propósito en la vida $(6,11,15,16$, 20), y crecimiento personal $(21,26,27,28)$.

Modelo en población universitaria chilena. El modelo 11 contempla seis factores de primer orden retomando 37 ítems (eliminando los reactivos 10 y 34) (Veliz, 2012).

Modelos en población mexicana. El modelo 12 postula cuatro factores, distribuyendo 34 ítems divididos en: 1) autoaceptación $(1,6,7,11,12,16,17,18,19,21,23,24$, $28,29,31)$; 2) relaciones interpersonales $(2,5,8,14,20,26$, $32,37)$; 3) autonomía $(4,9,13,22,25,33)$, y 4) satisfacción con la vida $(30,35,36,38,39)$ (Loera et al., 2008).

El modelo 13 incluye dos factores: 1) bienestar positivo, integrado por 14 reactivos $(1,4,5,8,9,10,11$, $12,13,15,16,18,19,20)$, y 2) bienestar negativo, con seis ítems $(2,3,6,7,14,17)$ (Lozano y García, 2016).

La prueba de los modelos se efectuó con 39 y 29 ítems conforme a la reducción propuesta por Díaz et al. (2006), mediante el método de estimación de máxima verosimilitud, usándose los estadísticos de bondad de ajuste más ampliamente recomendados en la literatura ( $\mathrm{Hu}$ y Bentler, 1999): chi cuadrado ( $\chi 2)$, grados de libertad (gl), tasa de ajuste $(\chi 2 / g l)$, índice de bondad de ajuste (GFI), índice de ajuste comparativo (CFI), índice Tucker-Lewis (TLI) y error de aproximación residual de la raíz de la media cuadrática (RMSEA). Como norma general, se establece un buen ajuste del modelo cuando: tasa $\chi 2 / g \mid \leq 3$; GFI, AGFI y $\mathrm{TLI}>0.90 ; \mathrm{CFI}>0.95 ;$ RMSEA $\leq 06$.

Finalmente se analizó la fiabilidad del modelo a partir del uso de los estadígrafos "alfa" de Cronbach y "Omega", de acuerdo con autores como Ventura y Caycho (2017), quienes refieren que valores superiores a 0.75 indican adecuados niveles de confiabilidad.

Todos los análisis de datos fueron hechos con los software SPSS 25 y AMOS 21.

\section{Resultados}

Una vez recabados los datos se hizo la limpieza de éstos; se recibieron 1,481 encuestas, de las cuales 41 se eliminaron debido a la presencia de valores perdidos y 40 por mostrar respuestas no comprometidas, concretándose así una muestra final de 1,400 casos; una vez efectuado este procedimiento, se hizo el análisis factorial confirmatorio de los modelos planteados.

\section{Análisis de Modelos}


Con la finalidad de probar los modelos propuestos en Los índices de ajuste encontrados en los 13 modelos con 39 y 29 reactivos se muestran en la tabla 1 . Como se puede observar, los 13 modelos analizados con 39 reactivos presentan índices de ajuste insatisfactorios, por lo que se procedió al análisis con 29 reactivos, donde se encontró que los modelos 8 y 9 propuestos obtuvieron buenos valores de ajuste, aproximados a los establecidos por autores como Hu y Bentler (1999), por lo cual se retomó el modelo 8 por su estructura más simple bajo el criterio de parsimonia, para ser revaluado.

\section{Tabla 1}

Índices de ajuste de los modelos cotejados.

\begin{tabular}{|c|c|c|c|c|c|c|c|}
\hline \multicolumn{8}{|c|}{$\begin{array}{l}\text { ANÁLISIS FACTORIAL CONFIRMATORIO } 39 \text { REACTIVOS EN } \\
\text { ESTUDIANTES UNIVERSITARIOS }\end{array}$} \\
\hline Modelo & $\times 2$ & $\mathrm{GL}$ & X2/GL & CFI & TLI & GFI & RMSEA \\
\hline $\begin{array}{l}\text { Modelo } \\
1\end{array}$ & 3431.394 & 702 & 4.888 & .708 & .692 & .707 & .075 \\
\hline $\begin{array}{l}\text { Modelo } \\
2\end{array}$ & 2319.743 & 701 & 3.309 & .796 & .784 & .841 & .057 \\
\hline $\begin{array}{l}\text { Modelo } \\
3\end{array}$ & 2953.329 & 692 & 4.268 & .715 & .695 & .761 & .068 \\
\hline $\begin{array}{l}\text { Modelo } \\
4\end{array}$ & 2946.442 & 687 & 4.289 & .715 & .693 & .761 & .069 \\
\hline $\begin{array}{l}\text { Modelo } \\
5\end{array}$ & 2994.576 & 697 & 4.296 & .711 & .692 & .752 & .069 \\
\hline $\begin{array}{l}\text { Modelo } \\
6\end{array}$ & 3000.448 & 696 & 4.311 & .710 & .691 & .751 & .069 \\
\hline $\begin{array}{l}\text { Modelo } \\
7\end{array}$ & 2980.756 & 695 & 4.289 & .712 & .693 & .758 & .069 \\
\hline $\begin{array}{l}\text { Modelo } \\
8\end{array}$ & 1443.906 & 269 & 5.368 & .767 & .740 & .828 & .079 \\
\hline $\begin{array}{l}\text { Modelo } \\
9\end{array}$ & 1445.170 & 271 & 5.333 & .767 & .742 & .828 & .079 \\
\hline $\begin{array}{l}\text { Modelo } \\
10\end{array}$ & 575.752 & 113 & 5.095 & .806 & .766 & .899 & .077 \\
\hline $\begin{array}{l}\text { Modelo } \\
11\end{array}$ & 2675.257 & 614 & 4.357 & .733 & .710 & .773 & .069 \\
\hline $\begin{array}{l}\text { Modelo } \\
12\end{array}$ & 2283.092 & 521 & 4.382 & .755 & .737 & .815 & .070 \\
\hline $\begin{array}{l}\text { Modelo } \\
13\end{array}$ & 1175.523 & 169 & 6.956 & .717 & .682 & .825 & .092 \\
\hline \multicolumn{8}{|c|}{$\begin{array}{c}\text { ANÁLISIS FACTORIAL CONFIRMATORIO } 29 \text { REACTIVOS EN } \\
\text { ESTUDIANTES UNIVERSITARIOS }\end{array}$} \\
\hline MODELO & $\times 2$ & $\mathrm{GL}$ & X2/GL & CFI & TLI & GFI & RMSEA \\
\hline $\begin{array}{l}\text { Modelo } \\
1\end{array}$ & 1972.455 & 377 & 5.232 & .710 & .688 & .801 & .078 \\
\hline
\end{tabular}

\begin{tabular}{|c|c|c|c|c|c|c|c|}
\hline \multicolumn{8}{|c|}{$\begin{array}{c}\text { ANÁLISIS FACTORIAL CONFIRMATORIO } 29 \text { REACTIVOS EN } \\
\text { ESTUDIANTES UNIVERSITARIOS }\end{array}$} \\
\hline MODELO & $\times 2$ & $\mathrm{GL}$ & $\times 2 / G L$ & CFI & TLI & GFI & RMSEA \\
\hline $\begin{array}{l}\text { Modelo } \\
2\end{array}$ & 1340.695 & 376 & 3.566 & .825 & .841 & .876 & .061 \\
\hline $\begin{array}{l}\text { Modelo } \\
3\end{array}$ & 1516.946 & 367 & 4.133 & .791 & .769 & .848 & .067 \\
\hline $\begin{array}{l}\text { Modelo } \\
4\end{array}$ & 1493.306 & 363 & 4.114 & .795 & .770 & .852 & .067 \\
\hline $\begin{array}{l}\text { Modelo } \\
5\end{array}$ & 1560.762 & 372 & 4.196 & .784 & .764 & .839 & .068 \\
\hline $\begin{array}{l}\text { Modelo } \\
6\end{array}$ & 1583.545 & 371 & 4.268 & .780 & .759 & .837 & .068 \\
\hline $\begin{array}{l}\text { Modelo } \\
7\end{array}$ & 1478.957 & 343 & 4.312 & .785 & .763 & .846 & .069 \\
\hline $\begin{array}{l}\text { Modelo } \\
8\end{array}$ & 523.263 & 129 & 4.056 & .884 & .862 & .922 & .066 \\
\hline $\begin{array}{l}\text { Modelo } \\
9\end{array}$ & 527.365 & 131 & 4.026 & .883 & .864 & .922 & .066 \\
\hline $\begin{array}{l}\text { Modelo } \\
10\end{array}$ & 575.752 & 113 & 5.095 & .806 & .766 & .899 & .077 \\
\hline $\begin{array}{l}\text { Modelo } \\
11\end{array}$ & 1492.604 & 362 & 4.123 & .795 & .770 & .852 & .067 \\
\hline $\begin{array}{l}\text { Modelo } \\
12\end{array}$ & 1257.526 & 293 & 4.292 & .808 & .787 & .809 & .069 \\
\hline $\begin{array}{l}\text { Modelo } \\
13\end{array}$ & 919.271 & 118 & 7.790 & .742 & .703 & .847 & .099 \\
\hline
\end{tabular}

Nota: $X 2=$ chi cuadrado; gl=grados de libertad, tasa de ajuste $(X 2 / g l)$, índice de bondad de ajuste (GFI), índice de ajuste comparativo (CFI), índice Tucker-Lewis (TLI) y error de aproximación residual de la raíz de la media cuadrática (RMSEA).

Para continuar con el análisis, en la submuestra dos se examinó el modelo 8 y se procedió a efectuar las modificaciones propuestas de índices de modificación, donde se correlacionaron los errores de medición de los ítems 1 y 7 , y 7 y 19 del área de autoaceptación; el 5 y el 22 del área dominio del entorno; el 24 y 37, y el 37 y 38 de la dimensión de crecimiento personal; y finalmente los reactivos 6 y 12, y 6 y 23 de propósito en la vida, mejorando sus índices de ajuste (tabla 2). 


\section{Tabla 2}

Índices de ajuste de los modelos de bienestar para universitarios mexicanos.

\begin{tabular}{|c|c|c|c|c|c|c|c|}
\hline MOdelo & $\times 2$ & $\mathrm{GL}$ & $\begin{array}{c}\times 2 / \\
\mathrm{GL}\end{array}$ & CFI & TLI & GFI & RMSEA \\
\hline $\begin{array}{l}\text { Modelo } \\
4 \\
\text { factores }\end{array}$ & 394.523 & 122 & 3.2 & .92 & .90 & .94 & .05 \\
\hline $\begin{array}{l}\text { Modelo } \\
6 \\
\text { factores }\end{array}$ & 652.240 & 170 & 3.8 & .90 & .90 & .92 & .06 \\
\hline
\end{tabular}

Nota: $X 2=$ chi cuadrado; $g$ l=grados de libertad, tasa de ajuste $(X 2 / g l)$, índice de bondad de ajuste (GFI), índice de ajuste comparativo (CFI), índice Tucker-Lewis (TLI) y error de aproximación residual de la raíz de la media cuadrática (RMSEA).

Aunado a lo anterior, a partir del análisis pormenorizado de la estructura original del instrumento y haciendo una exhaustiva exploración de los datos y eliminación de reactivos, se encontró un segundo modelo con buenas características de ajuste compuesto por seis dimensiones y 20 reactivos (tabla 2 ).

Los criterios para la eliminación de reactivos en este modelo corresponden a 1) el valor encontrado en la matriz de covarianzas de error estandarizado superiores a 6 , y 2) valor del peso factorial del reactivo hacia su dimensión, eliminándose así los reactivos 1 de autoaceptación, 14 y 32 del área relaciones positivas, el 3, 15 y 27 de autonomía, el 16 y 39 de dominio del entorno, y el 36 de conocimiento personal. Además se retomaron las propuestas del programa en índices de modificación en las covarianzas del error de medición de los reactivos 7 con 19, 4 con 9, 37 con 38 y 6 con 23 (figura 1 ).

\section{Análisis de fiabilidad}

A partir de los modelos confirmados se procedió a calcular los índices de fiabilidad alfa de Cronbach y el coeficiente omega (Ventura y Caycho, 2017); en la tabla 3 se muestran los valores de los coeficientes por dimensión de los modelos finales obtenidos.

\section{Tabla 3}

Coeficientes de fiabilidad alfa de Cronbach y Omega por escala de los modelos de 4 y 6 factores.

\begin{tabular}{|c|c|c|}
\hline $\begin{array}{l}\text { MODELO DE CUATRO FACTORES CON } \\
18 \text { REACTIVOS }\end{array}$ & Alfa & OMEgA \\
\hline Autoaceptación & .767 & .605 \\
\hline $\begin{array}{l}\text { 1. Cuando repaso la historia de mi } \\
\text { vida... }\end{array}$ & & \\
\hline
\end{tabular}

7. En general, me siento seguro...

\section{MOdelo de CUATRO FACTORES CON 18 REACTIVOS \\ Alfa \\ Omega}

19. Me gusta la mayor parte de...

31. En su mayor parte, me siento orgulloso...

Dominio del entorno

5. Me resulta difícil dirigir mi vida...

11. He sido capaz de construir un hogar...

16. En general, siento que soy responsable...

22. Las demandas de la vida...

39. Si me sintiera infeliz con mi situación...

Crecimiento personal

.629

.607

6. Disfruto haciendo planes...

12. Soy una persona activa...

17. Me siento bien cuando pienso...

18. Mis objetivos en la vida...

23. Tengo clara la dirección..

Propósito en la vida .783 .642

24. En general, con el tiempo siento...

36. Cuando pienso en ello...

37. Tengo la sensación de que...

38. Para mí, la vida ha sido un proceso...

Total

.892

.862

Modelo de SeIs factores con 20 Alfa OMEga
REACTIVOS

Autoaceptación

.767

.613

7. En general, me siento seguro...

19. Me gusta la mayor parte de...

31. En su mayor parte, me siento orgulloso...

Relaciones positivas

.672

.442

2. A menudo me siento solo...

8. No tengo muchas personas...

16. En general, siento que soy responsable.

Autonomía

.594

.468 

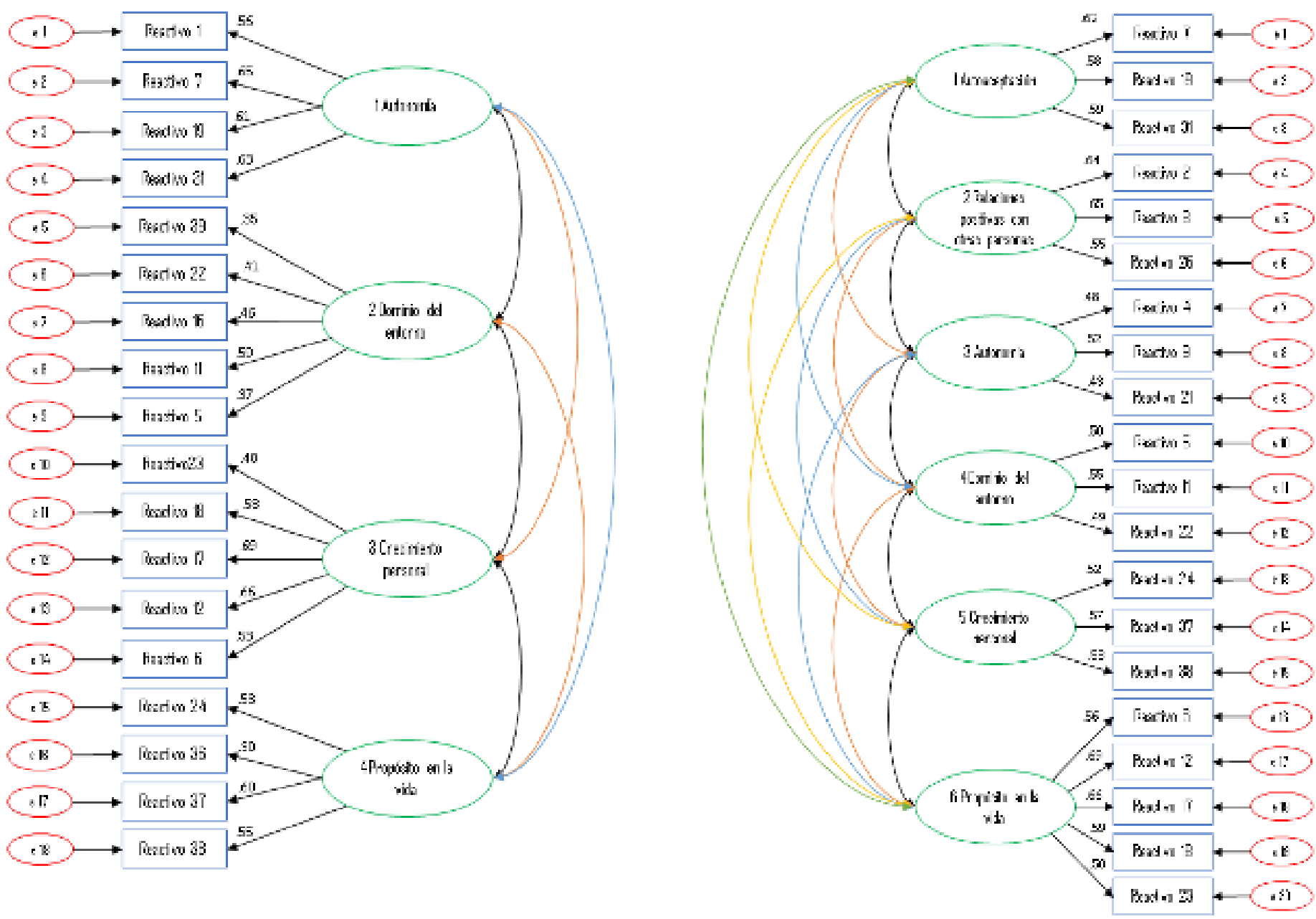

\section{Figura 1}

Comparación de modelos de la estructura factorial del Ryff con 4 y 6 dimensiones.

Modelo de seis factories con 20 Alfa OMEga
reactivos

4. Me preocupa cómo otra gente...

9. Tiendo a preocuparme...

21. Tengo confianza en mis opiniones...

Dominio del entorno .564 511

5. Me resulta difícil dirigir mi vida...

11. He sido capaz de construir un hogar...

22. Las demandas de la vida...

Crecimiento personal

.692

.511

24. En general, con el tiempo siento...

37. Tengo la sensación de que con el tiempo...

\section{MOdelo de SEIS FACTORES CON 20 REACTIVOS \\ Alfa

38. Para mí, la vida ha sido un proceso...

Propósito en la vida

.783

.754

6. Disfruto haciendo planes..

12. Soy una persona activa...

17. Me siento bien cuando pienso...

18. Mis objetivos en la vida...

23. Tengo clara la dirección...

Total

.899

.875

Como se muestra en la tabla 3 los índices de fiabilidad de la escala total de ambos modelos se indican aceptables; sin embargo, al hacer el análisis por escala la calidad de la fiabilidad observada se muestra moderada, 
siendo, en el modelo de cuatro reactivos, la dimensión de dominio del entorno la que presenta los valores más bajos, y en la escala de seis factores, autonomía y dominio del entorno presentan valores poco satisfactorios.

Por otra parte, la escala de propósito en la vida se muestra con coeficientes adecuados para ambos modelos. Se descarta la posibilidad de que se presente un incremento artificial del índice alfa a partir del número de reactivos dada su correspondencia con el índice omega.

\section{Discusión}

Las Escalas de Bienestar Psicológico (EBP) (Riff, 1989a), hoy son el instrumento más popular para hacer mediciones del bienestar; sin embargo hay controversia respecto a su estructura factorial, por lo que el objetivo de esta investigación fue analizar la validez de constructo de los principales modelos teóricos del bienestar propuestos en la literatura, para determinar cuál de ellos presenta los niveles de ajuste más satisfactorios en una muestra de universitarios mexicanos.

Después de someter los 26 modelos propuestos en la literatura consultada a un análisis meticuloso, se encontró que sólo el modelo 8 (Tomás et al., 2012) mostró índices de ajuste cercanos a valores satisfactorios propuestos por diferentes autores (Hu y Bentler, 1999; Furr, 2017); este modelo ha sido confirmado en la literatura como el núcleo del bienestar eudaimónico (Freire et al., 2017; Tomás et al., 2012). No obstante, este modelo de cuatro factores requirió de múltiples correlaciones, por lo que, aunque se reconocen índices de ajuste adecuados, en futuros análisis se propone examinar las implicaciones teóricas.

Por otra parte, a partir de los análisis y eliminación de reactivos en el modelo original de Ryff (1989a), surgió un modelo de seis dimensiones con 20 reactivos, con índices de ajuste satisfactorios, situación que implica correspondencia entre los datos empíricos de esta investigación y el modelo teórico del bienestar desde el enfoque paradigmático de la felicidad eudaimónica (Ryff, 1989a), por lo que se considera que esta estructura puede fungir como una versión alternativa breve para estudiantes universitarios mexicanos y facilitar su aplicación en diferentes ámbitos.

Es importante mencionar que ambas estructuras, pese a exponer modelos distintos, tienen índices de ajuste similares; los autores se decantan por el modelo de seis factores como el más indicado para evaluar los niveles del bienestar en universitarios, dada su correspondencia teórica (Ryff, 1989a), a pesar de ser menos parsimonioso (Cheung y Rensvold, 2001; Furr, 2017).
Aunado a lo anterior, a partir de los modelos confirmados se calcularon los índices de fiabilidad alfa de Cronbach y omega, los cuales son satisfactorios para el total de la escala en ambos modelos; sin embargo, al hacer el análisis por escala se presentan dimensiones cuyos coeficientes de fiabilidad se muestran moderados, inferiores a los identificados por otros autores en muestras mexicanas (Loera et al., 2008; Lozano y García, 2016; Medina et al., 2013), por lo que se sugiere hacer un análisis que revalide estos datos.

\section{CONCLUSIONES}

El bienestar psicológico es un término de interés para diversas disciplinas, incluida la Psicología; con frecuencia es asociado a conceptos como felicidad, calidad de vida, salud psicológica, salud biológica y buena situación económica.

Este constructo es relevante cuando se analizan diversos aspectos que influyen en el logro académico universitario porque su rendimiento es constantemente evaluado, tiene periodos amplios de clase, muchas horas de estudio, y en ocasiones relaciones conflictivas con docentes y coetáneos. Por lo anterior, es indispensable tener instrumentos válidos que permitan evaluarlo de manera precisa, para poder tener pautas e intervenir cuando la situación lo amerite.

A partir de los análisis y eliminación de reactivos acerca del modelo original de Ryff (1989a), este trabajo propone un modelo que incluye las seis dimensiones teóricas del constructo con 20 reactivos como una versión alternativa breve para evaluar el bienestar psicológico en estudiantes universitarios mexicanos de manera válida y confiable (apéndice).

A pesar de los resultados obtenidos, existen algunas consideraciones que deben considerarse en futuras investigaciones, como analizar la fiabilidad de cada una de las escalas teniendo en cuenta lo que mencionan Keyes et al. (2002) respecto a la necesidad de seleccionar/ eliminar determinados ítems para maximizar la validez de contenido, aunque esto vaya en detrimento de la consistencia interna (Keyes et al., 2002, p. 1001).

Para futuros estudios se propone también diversificar la muestra porque la presente sólo incluye participantes del centro de la República.

Por último, debido a la variabilidad encontrada en los modelos analizados por diferentes autores, se propone continuar con la exploración del constructo en diferentes muestras con el objetivo de probar la generalización de la solución factorial propuesta en este trabajo. 


\section{REFERENCIAS}

Abbott, R. A., Ploubidis, G. B., Huppert, F. A., Kuh, D. \& Croudace, T. J. (2010). An evaluation of precision of mea-surement of Ryff's Psychological Well-Being Scales in apopulation sample. Social Indicators Research, 97(3), 357 373. http:// dx.doi.org/10.1007/s11205-009-9506-x

Abbott, R. A., Ploubidis, G.B., Huppert, F.A., Kuh, D., Wadsworth, M.E.J. \& Croudace TJ. (2006). Psychometric evaluation and predictive validity of Ryff's psychological well-being items in a UK birth cohort sample of women. Health and Quality of Life Outcomes, 4, 76. http:// doi.org/10.1186/14777525-4-76

Caballero, C.; Bresó, E., \& González G. (2015). Burnout en estudiantes universitarios [Burnout in university students]. Psicología desde el Caribe, 32(3), 424-441.

Carlotto, M., Gonçalves-Câmara, S. \& Brazil-Borges, Á. (2005). Predictores del Síndrome de Burnout en estudiantes de un curso técnico de enfermería. Diversitas: Perspectivas en Psicología, 1 (2), 195-204.

Chen, F. F., Jing, Y., Hayes, A., \& Lee, J.M. (2013). Two concepts or two approaches? A bifactor analysis of psychological and subjective well-being. Journal of Happiness Studies, 14 (3), 1033-1068. http://dx.doi.org/10.1007/s10902-012-9367-x

Cheung, G.W., \& Rensvold, R.B. (2001). The effects of model parsimony and sampling error on the fi t of structural equation models. Organizational Research Methods, 4, 236-264. http://dx.doi. org/10.1177/109442810143004

Díaz, D., Rodríguez, R., Blanco, A., Moreno, B., Gallardo, I., Valle, C., et al., (2006). Adaptación española de las Escalas de Bienestar Psicológico de Ryff [Spanish adaptation of the Psychological Well-Being Scales]. Psicothema, 18(3), 572577.

Freire, A., Ferradás, M.M., Nuñez, J.C., \& Valle, A. (2017). Estructura factorial de las Escalas de Bienestar Psicológico de Ryff en estudiantes universitarios [The factorial structure of Ryff's Psychological Well-Being Scales in university studentsAbstract]. European Journal of Education and Psychology, 10, 1-8. http://dx.doi.org/10.1016/j. ejeps.2016.10.001

Furr, M.(2017). Psychometrics: An introduction. EUA: SAGE Publications.

García-Alandete, J. (2013). Bienestar psicológico, edad y género en universitarios españoles [Psychological well-being, age, and, gender among spanish undergraduates]. Salud \& Sociedad, 4(1), 48-58. http://dx.doi.org/10.22199/ S07187475.2013.0001.00004

Hu, L.T, \& Bentler, P.M.(1999). Cutoff criteria for fit indexes in covariance structure analysis: Conventional criteria versus new alternatives. Struct Equ Modeling, 6,1-55.

Kafka, G.J., \& Kozma, A. (2002). The construct validity of Ryff's scales of psychological well-being (SPWB) and their relationship to measures of subjective well-being. Social Indicators Research; 57(2): 171-190. http://dx.doi. org/10.1023/A:10144517252040

Kállay E, Rus C. (2014). Psychometric properties of the 44-item version of Ryff's Psychological Well-Being Scale. European Journal of Psychological Assessment, 30(1), 15-21. http:// dx.doi.org/10.1027/1015-5759/a000163

Keyes, C.L.M., Shmotkin, D. \& Ryff, C. D. (2002). Optimizingwellbeing: The empirical encounter of two traditions. Jour-nal of Personality and Social Psychology, 82(6), 1007-1022. http://dx.doi.org/10.1037//0022-3514.82.6.1007

Loera, M.N., Balcázar, N.P., Trejo, G.L., Gurrola, P.G., \& Bonilla, M.M. (2008). Adaptación de la escala de bienestar psicológico de Ryff en adolescentes preuniversitarios [Adaptation of the Ryff's scale of psychological wellbeing in pre-university adolescents]. Revista Neurología, Neurocirugía y Psiquiatría, 41(3-4), 90-97.

Lozáno, A.M., \& García, G.B. (2016). Escala de Bienestar Psicológico (EBP). In B García, M Lozano, \& I Díaz, (Eds). 19 escalas de evaluación psicológica (pp.77-81). México

Martínez, I. \& Marques, A. (2005). Burnout en estudiantes universitarios de España y Portugal y su relación con variables académicas [Burnout in university students and their relationship with academic variables]. Aletheia, 21, 21-30.

Medina, C.M., Gutiérrez, H.C., \& Padrós, B.F. (2013). Propiedades psicométricas de la escala de bienestar psicológico de Ryff en población mexicana [Psychometric Properties of Ryff's Scale of Psychological Well-being in a Mexican Population]. Revista de Educación y Desarrollo, 27, 25-30.

Rodríguez, R., Díaz, D., Moreno, B., Blanco, A., \& Van Dierendonk, D. (2010). Vitalidad y recursos internos como componentes del constructo de bienestar psicológico [Vitality and inner resources as relevant components of psychological wellbeing]. Psicothema, 22, 63-70.

Ryan, R., \& Huta, V. (2009). Wellness as healthy functioning or wellness as happiness: the importance of eudaimonic thinking (response to the Kashdan et al. and Waterman discussion). The Journal of Positive Psychology, 4( 3), 202 204.

Ryff, C.D. (1989a). Happiness is Everything, or is It? Explorations on the Meaning of Psychological Well-Being. Journal of Personality and Social Psychology, 57, 1069-1081. http:// dx. doi:10.1037//0022-3514.57.6.1069

Ryff, C.D. (1989b). Beyond Ponce de Leon and Life Satisfaction: New Directions in Quest of Successful Aging. International Journal of Behavioral Development, 12, 35-55. http://dx. doi:10.1177/016502548901200102

Ryff, C.D., \& Keyes, C.L.M. (1995). The Structure of Psychological Well-Being Revisited. Journal of Personality and Social Psychology, 69(4), 719-727. http:// dx.doi:10.1037//0022-3514.69.4.719

Ryff, C.D., \& Singer, B. H. (2008). Know Thyself and Become What You Are: A Eudaimonic Approach to Psychological WellBeing. Journal of Happiness Studies, 9(1), 13-39. http:// dx.doi:10.1007/s10902-006-9019-0

Springer, K. W., \& Hauser, R. M. (2006). An assessment of theconstruct validity of Ryff's Scales of Psychological Well-being: Method, mode and measurement effects. Social Science Research, 35(4), 1080-1102. http://dx.doi. org/10.1016/j.ssresearch.2005.07.004

Streiner, D. L., Norman, G. R. \& Cairney, J. (2015). Health measurement scales, a practical guide to their deveopment and use. Oxford: United Kingdom.

Tomás, J.M., Meléndez, J.C., \& Navarro, E. (2008). Modelos 
factoriales confirmatorios de las escalas de Ryff en una muestra de personas mayores [Factorial confirmatory models of Ryff 's scales in a sample of elderly people]. Psicothema. 20 (2): 304-310.

Tomás, J.M., Sancho, P., Meléndez, J.C., \& Mayordomo, T. (2012). Resilience and coping as predictors of general well-being in the elderly: A structural equation modelling approach. Aging \& Mental Health. 2012; 16(3): 317-326. http:// dx.doi:10.1080.13607863.2011.615737.

Van Dierendonck, D. (2004). The construct validity of Ryff's scale of psychological well-being and its extension with spiritual well-being. Personality and Individual Differences, 36 (3), 629-643. https://doi.org/10.1016/S0191-8869(03)00122-3

Van Dierendonck, D., Díaz, D., Rodríguez-Carvajal, R., Blanco, A., \& Moreno-Jiménez, B. (2008). Ryff's six factor model of psychologial well-being, a Spanish exploration. Social Indicators Research, 87, 473-479. https://doi.org/10.1007/ s11205-007-9174-7

Veliz, A. (2012). Propiedades psicométricas de la escala de bienestar psicológico y su estructura factorial en universitarios chilenos [Psychometric properties and factor structure of scale of psychological well-being in chilean university students]. Psicoperspectivas, 11(2), 143-163.
Ventura, J.L., \& Caycho, T. (2017). El coeficiente Omega: un método alternativo para la estimación de la confiabilidad. Revista Latinoamericana de Ciencias Sociales, Niñez y Juventud, 15(1), 625-627.

Vera-Villarroel P, Urzúa MA, Silva J, Pavez P, \& Celis-Atenas K. (2013). Escala de Bienestar de Ryff: Análisis Comparativo de los Modelos Teóricos en Distintos Grupos de Edad [Ryff scale of well-being: factorial structure of theoretical models in different age groups]. Psicologia: Reflexão e Crítica, 26 (1), 106-112. http://dx.doi.org/10.1590/S010279722013000100012

Vielma, J, \& Alonso, L (2010). El estudio del bienestar psicológico subjetivo. Una breve revisión teórica [Studying Subjective Psychological Well-being. A literature review]. Educere. 14 (49): 265-275. 


\section{Escalas de bienestar psicológico*}

\section{Autoaceptación (7, 19, 31)}

7. En general, me siento seguro y positivo conmigo mismo. 19. Me gusta la mayoría de los aspectos de mi personalidad.

31. En su mayor parte, me siento orgulloso de quien soy y la vida que llevo.

\section{Relaciones positivas $(2,8,26)$}

2. A menudo me siento solo porque tengo pocos amigos íntimos con quienes compartir mis preocupaciones.

8. No tengo muchas personas que quieran escucharme cuando necesito hablar.

26. No he experimentado muchas relaciones cercanas y de confianza.

\section{Autonomía $(4,9,21)$}

4. Me preocupa cómo otra gente evalúa las elecciones que he hecho en mi vida.

9. Tiendo a preocuparme de lo que otra gente piensa de mí.

21. Tengo confianza en mis opiniones, incluso si son contrarias al consenso general.

\section{Dominio del entorno $(5,11,22)$}

5. Me resulta difícil dirigir mi vida hacia un camino que me satisfaga.

11. He sido capaz de construir un hogar y un modo de vida a mi gusto.

22. Las demandas de la vida diaria a menudo me deprimen.

\section{Crecimiento personal $(24,37,38)$}

24. En general, con el tiempo siento que sigo aprendiendo más acerca de mí mismo.

37. Tengo la sensación de que con el tiempo me he desarrollado mucho como persona.

38. Para mí, la vida ha sido un proceso continuo de estudio, cambio y crecimiento.

\section{Propósito en la vida $(6,12,17,18,23)$}

6. Disfruto haciendo planes para el futuro y trabajar para hacerlos realidad.

12. Soy una persona activa al realizar los proyectos que propuse para mí mismo.

17. Me siento bien cuando pienso en lo que he hecho y lo que espero hacer.

18. Mis objetivos en la vida han sido más una fuente de satisfacción que de frustración para mí.

23. Tengo clara la dirección y el objetivo de mi vida.

\section{* Los ítems inversos se presentan en cursivas.}




\section{Meta-Análisis del Artículo}




\section{Dimensión Cuantitativa}

\section{Perfil de Evaluación entre pares}
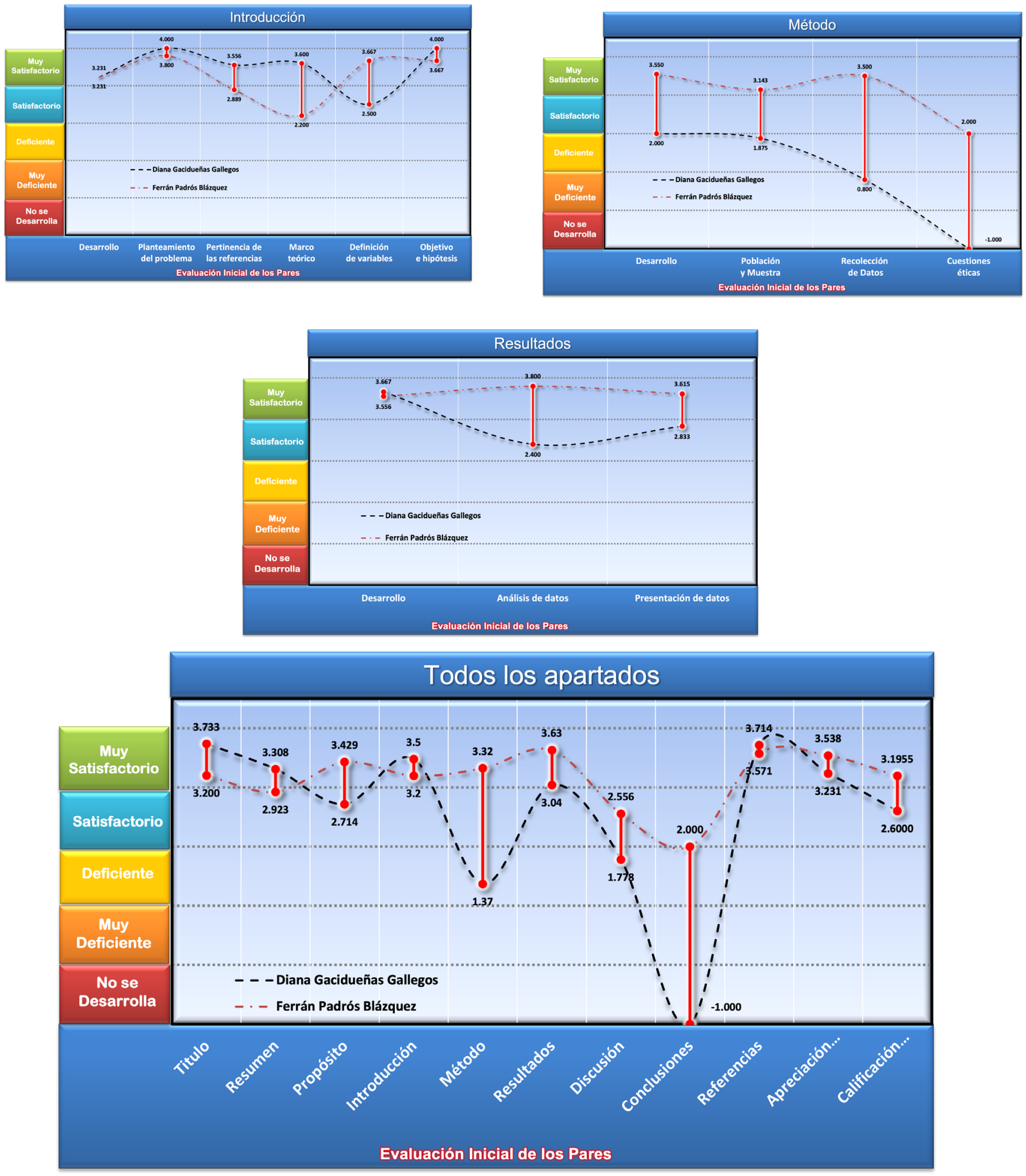


\section{Índice de Concordancia}

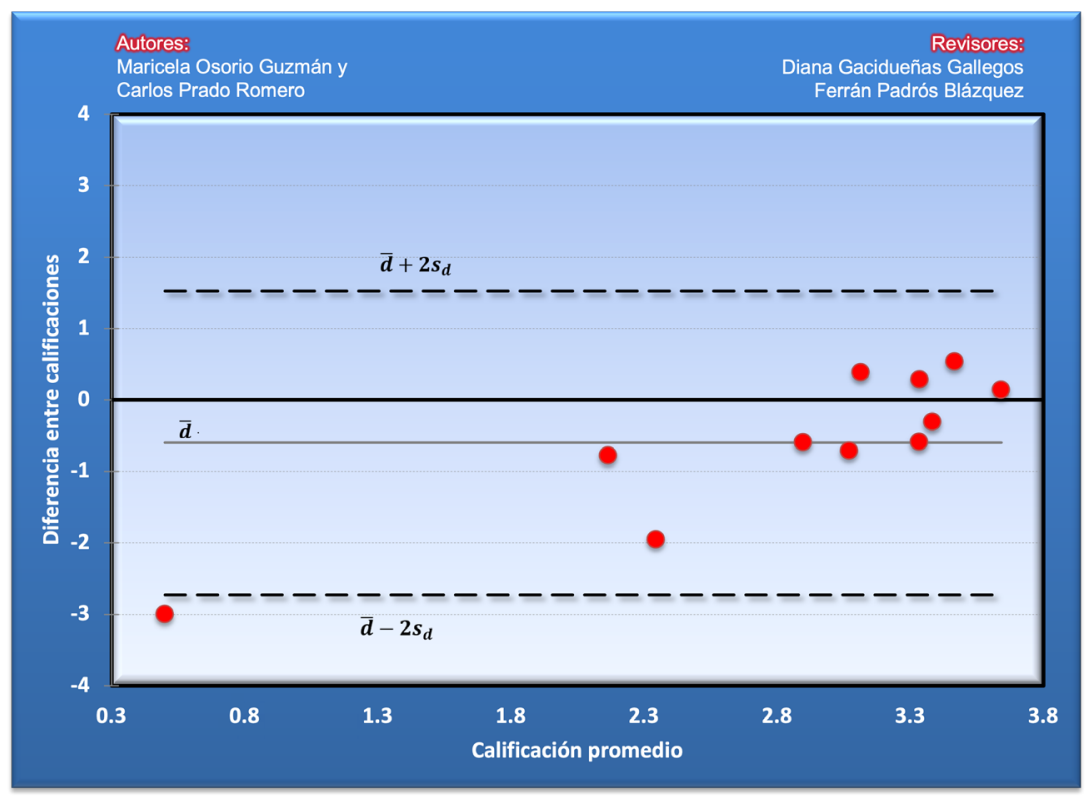

\section{Índice de Acuerdo}

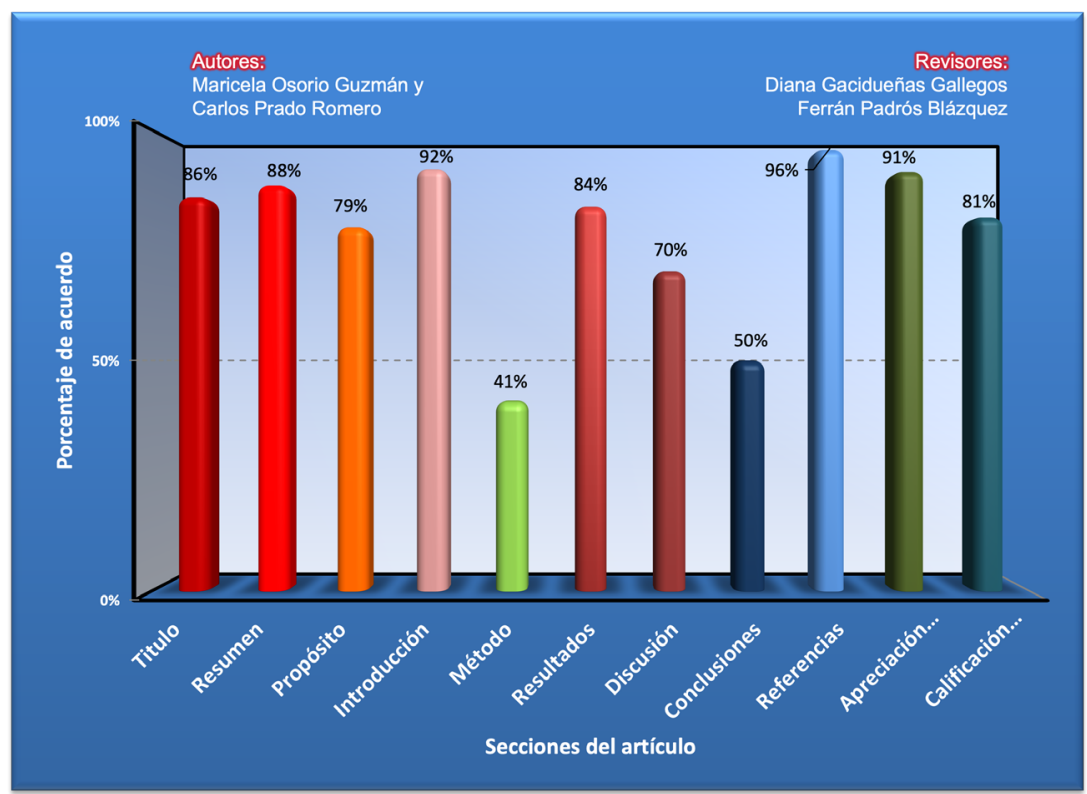




\begin{tabular}{|c|c|}
\hline Revisor 1 & Revisor 2 \\
\hline Diana Garcidueñas Gallegos & Ferran Padrós Blázquez \\
\hline \multicolumn{2}{|c|}{ Título/Autoría } \\
\hline $\begin{array}{l}\text { "en universitarios mexicanos" en lugar de "en una muestra } \\
\text { de universitarios mexicanos" }\end{array}$ & $\begin{array}{l}\text { Al título le sobra una palabra para tener } 15 \text { las que reco- } \\
\text { mienda la APA. Por otro lado, falta el correo electrónico } \\
\text { del coautor. En general está correcto. }\end{array}$ \\
\hline \multicolumn{2}{|c|}{ Resumen } \\
\hline $\begin{array}{l}\text { Resaltar el análisis factorial confirmatorio y sus índices } \\
\text { de ajuste en resultados, más allá de los índices de con- } \\
\text { fiabilidad. Por el número de palabras se podrían acotar } \\
\text { los antecedentes. Incluir en las palabras clave la pobla- } \\
\text { ción de estudio. }\end{array}$ & $\begin{array}{l}\text { En general es correcto, pero se observan algunos errores } \\
\text { de redacción: Sugiero revisar ...Esta escala, se ha usado en } \\
\text { países, muestras etarias... (debería poner usado en DIFE- } \\
\text { RENTES países... También revisar el sentido de la siguien- } \\
\text { te frase: ...por lo que su validez ha sido puesta a prueba } \\
\text { debido a que las soluciones factoriales varían considera- } \\
\text { blemente. El resumen tiene } 197 \text { palabras y se solicitan un } \\
\text { máximo de } 150 \text {, y se ofrecen } 4 \text { palabras clave en lugar de } \\
\text { 5. Alguna como el apellido de RYFF o el EFC no los con- } \\
\text { sidero muy pertinentes. }\end{array}$ \\
\hline \multicolumn{2}{|c|}{ Próposito del Estudio } \\
\hline $\begin{array}{l}\text { El propósito del estudio es claro, analizar los distintos } \\
\text { modelos existentes en la literatura respecto a la escala } \\
\text { de bienestar de Ryff, para determinar cuál presenta un } \\
\text { mejor ajuste con los datos de la muestra de estudiantes } \\
\text { universitarios mexicanos. Sin embargo, a partir del mé- } \\
\text { todo, se pierde un poco el hilo del propósito del estu- } \\
\text { dio. Los modelos se describen muy someramente, no se } \\
\text { reportan sus índices de ajuste previos. En la discusión } \\
\text { faltan más elementos que discutan la falta de ajuste con } \\
\text { los otros modelos. }\end{array}$ & En general esta sección es satisfactoria \\
\hline \multicolumn{2}{|c|}{ Introducción } \\
\hline $\begin{array}{l}\text { Se describe muy poco el marco teórico de la escala, sólo } \\
\text { se nombran las dimensiones pero no se explica su defini- } \\
\text { ción y la relación entre ellas. Resaltar la importancia de la } \\
\text { aplicación en esa muestra, se mencionan algunas variables } \\
\text { pero no se establece la relación de por qué evaluar el bien- } \\
\text { estar. Resultados de los otros estudios en cuanto al aná- } \\
\text { lisis psicométrico de la escala en esta población y en qué } \\
\text { poblaciones se probaron los otros modelos (¿eso podría } \\
\text { afectar en el ajuste?) }\end{array}$ & $\begin{array}{l}\text { El artículo, en general es de alta calidad y es muy per- } \\
\text { tinente. Sin embargo, en la introducción se detectan } \\
\text { algunos errores de redacción, se sugiere realizar una } \\
\text { revisión. Por ejemplo; "Debido al interés de esta inves- } \\
\text { tigación y tomado en cuenta lo mencionado por Freire } \\
\text { et al., en 2017; cuando refieren que son pocos los estu- } \\
\text { dios que prueben diferentes modelos estructurales del } \\
\text { bienestar psicológico en población universitaria; debi- } \\
\text { do también..." También se echa de menos profundizar } \\
\text { en el modelo teórico de Ryff, está poco desarrollado. }\end{array}$ \\
\hline
\end{tabular}




\begin{tabular}{|c|c|}
\hline Revisor 1 & Revisor 2 \\
\hline \multicolumn{2}{|c|}{ Método } \\
\hline $\begin{array}{l}\text { Describir con mayor detalle el procedimiento realiza- } \\
\text { do para conseguir los datos, sólo se menciona "se les } \\
\text { instó a firmar el consentimiento informado", pero no } \\
\text { se mencionan otras consideraciones éticas para llevar a } \\
\text { cabo el levantamiento de información. No se reportan } \\
\text { las características del instrumento en la sección corres- } \\
\text { pondiente, número de reactivos, opciones específicas } \\
\text { de respuesta, propiedades psicométricas reportadas, } \\
\text { versión española del instrumento. Especificación del } \\
\text { tamaño de la muestra obtenida, características de las } \\
\text { submuestras. Especificación de los datos sociodemo- } \\
\text { gráficos solicitados. Falta una mayor descripción de } \\
\text { los procedimientos estadísticos, una parte está fuera de } \\
\text { esta sección. }\end{array}$ & $\begin{array}{l}\text { En general el método es adecuado, pero se sugiere des- } \\
\text { cribir con mayor detalle en qué consisten los factores que } \\
\text { contempla el instrumento. El apartado de procedimiento, } \\
\text { se debería explicar cómo se obtuvo el consentimiento in- } \\
\text { formado (aunque se infiere) y sobre todo, si el protocolo } \\
\text { de investigación fue evaluado por un comité de ética. Así } \\
\text { como aclarar que la participación fue voluntaria y se pre- } \\
\text { servó el anonimato. }\end{array}$ \\
\hline \multicolumn{2}{|c|}{ Resultados } \\
\hline $\begin{array}{l}\text { Falta reportar los pesos factoriales, el porcentaje de va- } \\
\text { rianza, las correlaciones de los factores de los modelos } \\
\text { resultantes para que puedan ser analizados, se pueden in- } \\
\text { cluir en los gráficos para un análisis visual. }\end{array}$ & $\begin{array}{l}\text { El apartado de resultados está muy bien elaborado en } \\
\text { general. Sin embargo, se alude a un análisis factorial ex- } \\
\text { ploratorio que realizan con la mitad de la muestra y no } \\
\text { describen los hallazgos de forma adecuada. }\end{array}$ \\
\hline \multicolumn{2}{|c|}{ Discusión } \\
\hline $\begin{array}{l}\text { Argumento del por qué no pudo haber resultado el } \\
\text { ajuste con los otros modelos. Hipótesis alternativas res- } \\
\text { pecto a los resultados obtenidos en el ajuste del modelo } \\
8 \text { y el modelo nuevo con eliminación de reactivos. Dis- } \\
\text { cutir las limitaciones del estudio. }\end{array}$ & $\begin{array}{l}\text { Se sugiere profundizar en la discusión, tratar de proponer } \\
\text { explicaciones por qué ajusta el modelo } 8 \text { y no los demás. } \\
\text { Además se sugiere señalar la limitaciones y sugerencias } \\
\text { concretas para futuras investigaciones. }\end{array}$ \\
\hline \multicolumn{2}{|c|}{ Conclusiones } \\
\hline $\begin{array}{l}\text { Hace falta una sección de conclusiones que interprete los } \\
\text { hallazgos con los objetivos, se hagan sugerencias basadas } \\
\text { en la experiencia de la investigación, contribución especí- } \\
\text { fica del estudio, deficiencias del estudio y cómo podrían } \\
\text { manejarse posteriormente. }\end{array}$ & $\begin{array}{l}\text { Se sugiere incorporar un apartado de conclusiones, o } \\
\text { incluir la información al final de la discusión. }\end{array}$ \\
\hline \multicolumn{2}{|c|}{ Referencias } \\
\hline $\begin{array}{l}\text { Publicaciones recientes del análisis o de la aplicación de } \\
\text { la escala en esa población, hay una de } 2017 \text { en población } \\
\text { mexicana que podría considerarse. }\end{array}$ & $\begin{array}{l}\text { Bastante menos del } 50 \% \text { de referencias pertenecen a los } \\
\text { últimos años. Sin embargo, según mi opinión, es lógico y } \\
\text { razonable. Las referencias son pertinentes y poner algu- } \\
\text { nas actualizadas sería forzoso y poco adecuado. Por otro } \\
\text { lado, sugiero revisar las referencias, debido que en alguna } \\
\text { han puesto el nombre abreviado de la revista o han puesto } \\
\text { un guión donde no debe ir. Pero en general el apartado es } \\
\text { correcto. }\end{array}$ \\
\hline
\end{tabular}




\section{Historia del Proceso}

\section{EDITORIAL}

